\title{
Solar ultraviolet B exposure and global variation in tuberculosis incidence: an ecological analysis
}

\author{
Tjarda M. Boere ${ }^{1,2}$, Douwe H. Visser ${ }^{3}$, A. Marceline van Furth ${ }^{4}$, Paul Lips ${ }^{5}$ and \\ Frank G.J. Cobelens ${ }^{1,6}$
}

Affiliations: ${ }^{1}$ Amsterdam Institute for Global Health and Development and Dept of Global Health, Academic Medical Centre, Amsterdam, The Netherlands. ${ }^{2}$ Faculty of Earth and Life Sciences, Dept of Health Sciences, Section of Infectious Diseases, Vrije Universiteit, Amsterdam, The Netherlands. ${ }^{3}$ Dept of Paediatrics, OLVG West Medical Centre, Amsterdam, The Netherlands. ${ }^{4}$ Dept of Paediatric Infectious Diseases and Immunology, VU Medical Centre, Amsterdam, The Netherlands. ${ }^{5}$ Dept of Internal Medicine, Endocrine section, VU Medical Centre, Amsterdam, The Netherlands. ${ }^{6} \mathrm{KNCV}$ Tuberculosis Foundation, The Hague, The Netherlands.

Correspondence: Frank G.J. Cobelens, Amsterdam Institute for Global Health and Development, PO Box 22700, 1100 DE Amsterdam, The Netherlands. E-mail: f.cobelensdaaighd.org

@ERSpublications

Variation in UV-B exposure may partly clarify previously unexplained global differences in TB incidence http://ow.ly/lrrl30aZiHN

Cite this article as: Boere TM, Visser DH, van Furth AM, et al. Solar ultraviolet B exposure and global variation in tuberculosis incidence: an ecological analysis. Eur Respir J 2017; 49: 1601979 [https://oi.org/ 10.1183/13993003.01979-2016].

ABSTRACT Epidemiological evidence supports vitamin D deficiency as a risk factor for tuberculosis. Differences in solar ultraviolet B (UV-B) exposure, the major source of vitamin D, might therefore partially explain global variation in tuberculosis incidence.

In a global country-based ecological study, we explored the correlation between vitamin D-proxies, such as solar UV-B exposure, and other relevant variables with tuberculosis incidence, averaged over the period 2004-2013.

Across 154 countries, annual solar UV-B exposure was associated with tuberculosis incidence. Tuberculosis incidence in countries in the highest quartile of UV-B exposure was 78\% (95\% CI 57-88\%, $\mathrm{p}<0.001)$ lower than that in countries in the lowest quartile, taking into account other vitamin D-proxies and covariates. Of the explained global variation in tuberculosis incidence, $6.3 \%$ could be attributed to variations in annual UV-B exposure. Exposure to UV-B had a similar, but weaker association with tuberculosis notification rates in the multilevel analysis with sub-national level data for large countries (highest versus lowest quartile $29 \%$ lower incidence; $\mathrm{p}=0.057$ ).

The potential preventive applications of vitamin D supplementation in high-risk groups for tuberculosis merits further investigation.

This article has supplementary material available from erj.ersjournals.com

Received: Oct 092016 | Accepted after revision: March 112017

Conflict of interest: Disclosures can be found alongside this article at erj.ersjournals.com 


\section{Introduction}

Tuberculosis continues to be a major cause of morbidity and mortality worldwide [1]. In 2014, 9.6 million incident cases and 1.5 million deaths were estimated globally [2]. Infection with Mycobacterium tuberculosis results in active disease or latent infection that might be reactivated at a later stage [3]. Recently, the latent-active dichotomy has been debated, suggesting rather a continuum along which controlled infection, subclinical bacterial multiplication and overt clinical disease represent different stages $[3,4]$. Co-infection with HIV, malnutrition and type 2 diabetes are known to drive this continuum towards clinical disease [5,6]. Vitamin D status has been identified as another possible factor in relation to disease progression, and treatment of vitamin $\mathrm{D}$ deficiency or vitamin $\mathrm{D}$ supplementation are being considered [7-11].

Vitamin D is mainly derived from cutaneous synthesis through solar ultraviolet B (UV-B) exposure, and partly from dietary sources [12-14]. Skin pigmentation reduces the amount of vitamin D that can be derived from sunlight exposure, but does not totally prevent vitamin D synthesis; thus, with high UV-B exposure, dark-skinned individuals can attain sufficient levels of vitamin D [7]. The biologically active form of vitamin D, 1,25-dihydroxyvitamin D, reduces intracellular growth of $M$. tuberculosis through the modulation of macrophage function $[13,14]$. Adequate vitamin D status is associated with increased innate immune response and reduced initial bacterial invasion $[10,13]$. Moreover, vitamin D metabolites can control the magnitude of the adaptive immune response to protect the host from excessive tissue damage at the site of infection [7, 15]. Ultraviolet radiation can also have a more direct effect on the immune response to bacterial infections, by suppressing adaptive immunity and supporting innate immune responses [7].

Tuberculosis (TB) incidence shows marked global variation. It is highest in resource-poor countries, probably reflecting the importance of socioeconomic factors such as crowding and malnutrition [6]. In addition, TB incidence correlates with the prevalence of HIV infection within a given population [16]. Nonetheless, part of the global variation in TB incidence remains unexplained [17]. We hypothesised that global variation in solar UV-B exposure, as a proxy for vitamin D status, is an additional determinant of TB incidence. We therefore explored the association of TB incidence rates with factors that affect vitamin D status, such as UV-B exposure, skin pigmentation [7] and dietary vitamin D intake [12], as well as factors known to be associated with TB incidence [6], such as HIV infection [12], body mass index [18], type 2 diabetes [6] and indicators of socioeconomic development [6].

\section{Methods}

\section{Study design and inclusion criteria}

This ecological study included all countries worldwide as the unit of analysis, using national population level data. Countries with population counts below one million, for which TB incidence rates might have been unstable, were excluded (table S1). In a secondary analysis, we added sub-national regions to the dataset for selected countries with large geographic latitude coverage (table S2). All analyses and variables were averaged over the period 2004-2013, unless specified otherwise.

\section{Data sources and definitions}

\section{Outcome measures}

The outcome variable in the primary analysis was the estimated country-specific TB incidence rate per year, including all pulmonary and extra-pulmonary forms of TB. The secondary outcome was the country-specific notification rate for new, i.e. not previously treated, pulmonary TB with bacteriological confirmation (i.e. by smear examination, culture or molecular assay). Both metrics were derived from the World Health Organization database (www.who.int/tb/country/data/download/en/). The incidence of TB cannot be measured directly, as it reflects reported TB cases, as well as diagnosed, but unreported and non-diagnosed cases. It is therefore estimated by the WHO for each country and each year based on notification data and results from TB prevalence surveys and/or expert opinion about the proportion of incident TB cases reported by National Tuberculosis Programmes (NTPs) [19].

Sub-national TB case notification data were derived from country reports, either from publicly accessible sources or through contacts with NTP officials (table S3). Sub-national case notification data were also restricted to bacteriologically confirmed new pulmonary $\mathrm{TB}$ cases, with the method of confirmation depending on the surveillance case definition in the country concerned (table S4).

Determinants and covariates

We included in our analyses solar UV-B exposure and other known determinants of vitamin D status (vitamin D-proxies), and potential confounding variables (covariates) in the association between vitamin D-proxies and TB incidence. 
Vitamin D-proxies

Solar UV-B radiation data were derived from the Helmholtz Centre for Environmental Research (UFZ) website (www.ufz.de/gluv/index.php?en=32435). The UFZ obtained UV-B data for the period October 2004 to January 2013 from the Ozone Monitoring Instrument on-board the NASA EOS Aura spacecraft [20]. The UFZ data takes factors such as altitude, ozone and cloud coverage into account, to measure the amount of UV-B that reaches the earth's surface accurately [21]. Data of mean annual UV-B and seasonality of UV-B levels were used in our analyses. Mean annual UV-B exposure was calculated as the average noontime UV-B level throughout the year $\left(\mathrm{J} \cdot \mathrm{m}^{-2} \cdot \mathrm{day}^{-1}\right)$. Seasonality of UV-B levels was expressed as the standard deviation of mean monthly values throughout the year [20].

Other vitamin D-proxies included the degree of urbanisation, fish consumption, food fortification policies, and the average degree of skin pigmentation. The level of urbanisation was used as a proxy for indoor crowding, and was defined as the percentage of the population residing in urban areas, as defined by national statistical offices. Information on urbanisation level per country was derived from the World Bank website (http://data.worldbank.org/indicator/SP.URB.TOTL.IN.ZS). Human food supply quantities of pelagic and demersal saltwater and freshwater fish in $\mathrm{kg}$ per capita per year from the period 2004-2011 were derived from the UN Food and Agriculture Organization (FAO) (http://faostat3.fao.org/ download/FB/FBS/E). Total fish consumption was calculated at a national population level as the sum of food supply quantity for each of the fish types [22]. Existence of vitamin D fortification policies per country (yes/no) was obtained from a 2006 review [23]. We calculated the average degree of skin pigmentation for each country, based on the 2006 WHO Solar Ultraviolet Radiation report [25] that categorised the population of each country proportionally into light, medium, or deep skin pigmentation, based on race or ethnicity information of each country, using the Fitzpatrick skin tone chart (table 1).

\section{Covariates}

Potential covariates that were analysed in determining the relationship between vitamin D-proxies and TB incidence included country-specific median age, male to female ratio, purchasing power parity-adjusted per capita gross domestic product (GDP), Human Development Index (HDI) ranking, proportion of incident TB patients co-infected with HIV, type 2 diabetes prevalence, population-mean body mass index (BMI), and alcoholism, expressed as 12-month relative prevalence estimates of alcohol use disorders. Table S5 lists additional information for each covariate.

\section{TABLE 1 Characteristics of included countries}

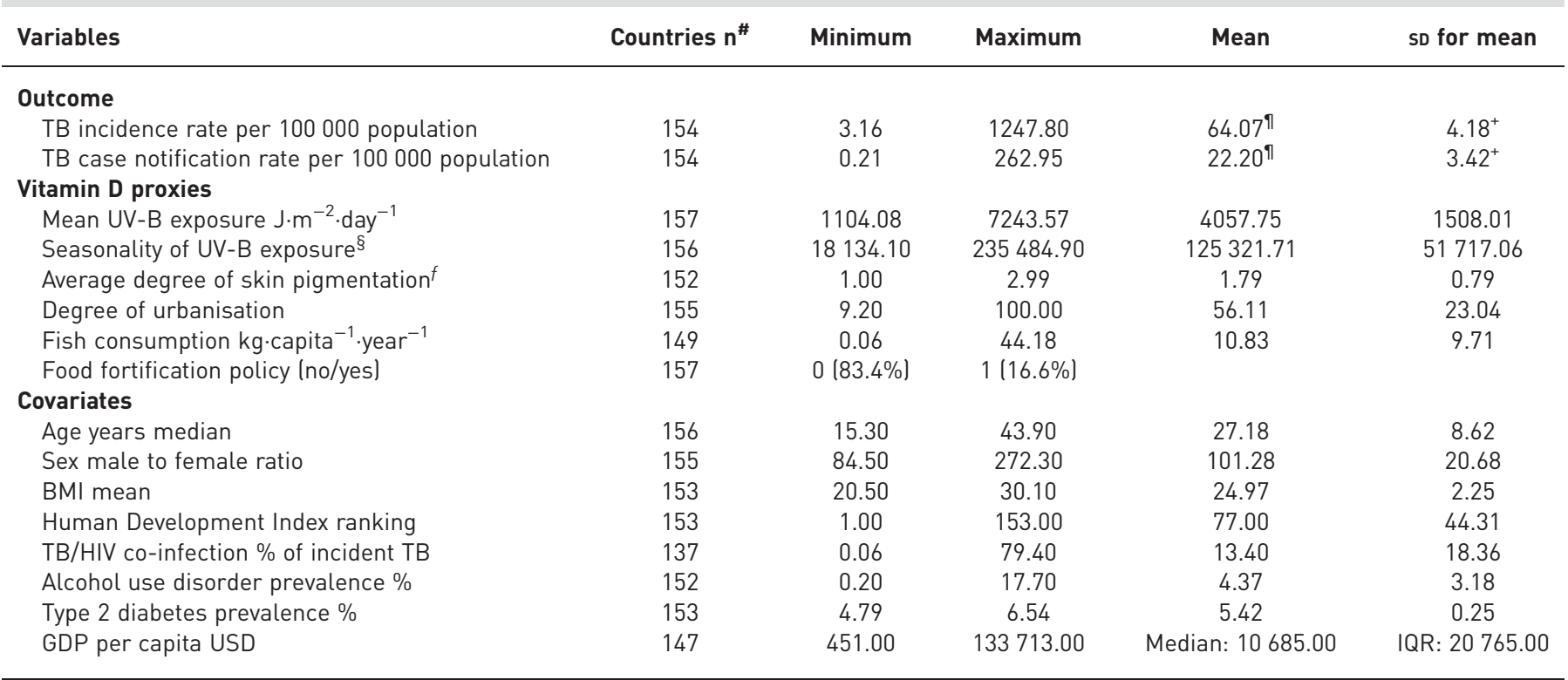

TB: tuberculosis; UV-B: ultraviolet B; BMI: body mass index; GDP: gross domestic product; IQR: interquartile range. ${ }^{\#}$ : for which data is available. ": geometric mean. ${ }^{+}$:SD for geometric mean. ${ }^{\S}$ : sD from mean monthly values $\left(\mathrm{J} \cdot \mathrm{m}^{-2}\right) .{ }^{f}$ : calculated for each country as follows: $((\%$ light skin pigmentation $/ 100) \times 1)+((\%$ medium skin pigmentation/100 $) \times 2)+((\%$ dark skin pigmentation $/ 100) \times 3)$. The average degree of skin pigmentation was categorised a priori as follows: 1.0-1.5 (category 1); $1.5-2.0$ (category 2); $2.0-2.5$ (category 3); 2.5-3.0 (category 4) for univariate and multivariate analyses. 


\section{Statistical analysis}

\section{Solar UV-B exposure}

The UFZ solar radiation data included UV-B levels referenced in the World Geodetic System 84 coordination system. A mean UV-B level was obtained for each country using the QGIS version 2.8.1 software (http://www.qgis.org/en/site/). First, UV-B data were added as a raster layer to the QGIS programme. Second, a world map of national and sub-national borders was added as a polygon vector layer. Third, zonal statistics were calculated to determine the UV-B levels per country. The UV-B seasonality data were derived similarly using the QGIS software. In a secondary analysis, sub-national UV-B data were used next to national level data for selected countries with large geographical coverage, following similar procedures. National and sub-national UV-B values were analysed using SPSS statistics version 20 (SPSS Inc., Chicago, IL, USA).

\section{Primary analysis}

We performed linear regression analyses in several steps. First, we checked conditions of normality and linearity, and log-transformed outcome measures if the assumption of normality was violated. Determinants that showed non-linear association with the outcome measure were ranked into quartiles or categorised otherwise. Skin pigmentation was categorised a priori, because the UV-B effect on vitamin D production decreases disproportionally with increased skin pigmentation [26]. Second, we checked bivariate analyses for high correlation among determinants and covariates, considering as a cut-off value, a Pearson's correlation of $\geqslant 0.90$. Third, we built a prediction model (vitamin D-proxy model) with vitamin D-proxies that showed significant association $(\mathrm{p}<0.05)$ with the outcome measure in univariate analysis. The "vitamin D-proxy model" was built using backward selection and a p-value of $>0.10$ to exclude variables. Next, we built a "fully adjusted model", in which covariates were checked for confounding effects on the vitamin D-proxy model. Covariates were included in the fully adjusted model if one of the regression coefficients changed by $\geqslant 10 \%$ after addition of the covariates, compared to the vitamin $\mathrm{D}$-proxy model. The proportion explained variation in per-country TB incidence that can be attributed to variation in solar UV-B exposure was calculated as the square of the adjusted partial correlation coefficient, controlling for other variables in the fully adjusted model.

\section{Secondary analyses}

Secondary analyses entailed national level linear regression analysis, and a multilevel linear regression model using country-level data, as well as sub-national level data for some of the larger, geographically varied countries. The multilevel model adjusted for clustering of sub-national level data within each corresponding country. By way of sensitivity analysis, both analyses considered as the outcome measure TB case notification rate instead of TB incidence. The procedure for building the fully adjusted model in the primary analysis was repeated for the secondary analyses, using the same vitamin D-proxy variables as in the primary vitamin D-proxy model. Sub-national level data included TB case notification rates, mean UV-B and seasonality of UV-B exposure, skin pigmentation, urbanisation, male to female ratio and HDI (table S3). Other variables were assumed to have homogeneous distribution throughout each country.

\section{Results}

Included in the study were 157 countries (tables 1, S1), covering $80.5 \%$ of all countries and territories worldwide. The TB incidence and case notification rates were available for 154 countries. The TB incidence rates ranged from 3.16 to $1247.80 / 100000$ per year and case notification rates ranged from 0.21 to $247.54 / 100000$ per year (table 1, figure S2, and table S6). Both outcome measures were log-transformed owing to violation of the normality assumption. Mean country-specific annual solar UV-B levels ranged from 1104.08 to $7243.57 \mathrm{~J} \cdot \mathrm{m}^{-2} \cdot$ day $^{-1}$ (table 1, figure 1). Urbanisation levels varied from $9.2 \%$ to $100 \%$. Both median age and per capita GDP were collinear with HDI ranking, and therefore excluded as covariates, as the HDI measure partially incorporates these factors in its calculation, and should thereby account for their effects. In univariate analyses (table 2), solar UV-B levels, seasonality of UV-B levels and fish consumption were non-linearly related to TB incidence rates, and thus ranked into quartiles. Body mass index was also non-linearly related to TB incidence, and therefore included as a categorical variable (table 1). Positive associations were noted between TB incidence and UV-B exposure, seasonality of UV-B exposure and skin pigmentation; whereas negative associations were noted between TB incidence and fish consumption, food fortification policy and urbanisation.

In the multivariable prediction model including vitamin D-proxies only, UV-B exposure, average skin pigmentation, urbanisation and fish consumption remained as predictors of TB incidence (table 3). The association between TB incidence and solar UV-B exposure was now reversed, showing a gradual decline in TB incidence with increasing levels of UV-B exposure. The HDI ranking and type 2 diabetes prevalence were identified as confounding covariates and were thus added to the model, resulting in the "fully 


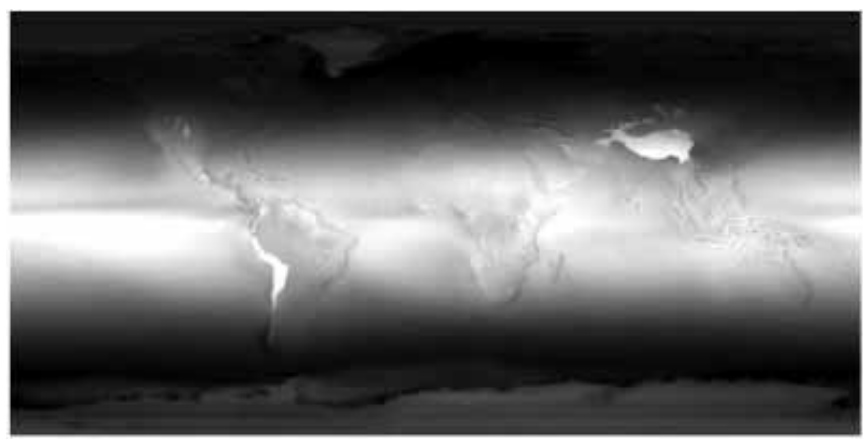

FIGURE 1 Mean annual solar ultraviolet B (UV-B) levels, 2004-2013. Worldwide distribution of mean annual solar UV-B levels. Illustration created after adding UV-B data as a raster layer to a world map of national and sub-national borders (polygon vector layer) in QGIS software (http://www.qgis.org/en/site/). Lighter colours correspond to higher mean annual solar UV-B levels. Data are derived from: www.ufz.de/gluv/index.php? en $=32435$.

adjusted model" (table 3). The reverse association between TB incidence and solar UV-B exposure was retained: countries in the highest UV-B quartile had $78 \%$ lower TB incidence ( $\beta$ coefficient 0.22 ) compared to countries in the lowest UV-B quartile, $(\mathrm{p}<0.001)$ (figure 2$)$. With a linear fit, the overall contribution of UV-B exposure remained significant in the fully adjusted model $(\mathrm{p}=0.016)$.

TABLE 2 Univariate associations between vitamin D proxies and covariates with tuberculosis (TB) incidence rates

\begin{tabular}{|c|c|c|}
\hline Variables & Regression coefficient ${ }^{\#}(95 \% \mathrm{Cl})$ & p-value \\
\hline \multicolumn{3}{|l|}{ Vitamin D proxies } \\
\hline \multicolumn{3}{|l|}{ Mean UV-B exposure $\mathrm{J} \cdot \mathrm{m}^{-2} \cdot$ day $^{-1 \uparrow}$} \\
\hline Second versus first quartile & $1.46(0.82-2.60)$ & 0.197 \\
\hline Third versus first quartile & $3.47(1.98-6.08)$ & $<0.001$ \\
\hline Fourth versus first quartile & $5.77(3.28-10.15)$ & $<0.001$ \\
\hline \multicolumn{3}{|l|}{ Seasonality of UV-B exposure $\mathrm{J} \cdot \mathrm{m}^{-2 \pi,+}$} \\
\hline Second versus first quartile & $0.46(0.25-0.86)$ & 0.014 \\
\hline Third versus first quartile & $0.41(0.22-0.75)$ & 0.004 \\
\hline Fourth versus first quartile & $0.26(0.14-0.48)$ & $<0.001$ \\
\hline Average degree of skin pigmentation & $2.16(1.89-2.46)$ & $<0.001$ \\
\hline Degree of urbanisation $\%$ & $0.96(0.95-0.97)$ & $<0.001$ \\
\hline \multicolumn{3}{|l|}{ Fish consumption kg.capita ${ }^{-1} \cdot \mathrm{year}^{-1 \uparrow}$} \\
\hline Second versus first quartile & $0.35(0.19-0.65)$ & 0.001 \\
\hline Third versus first quartile & $0.24(0.13-0.46)$ & $<0.001$ \\
\hline Fourth versus first quartile & $0.33(0.18-0.62)$ & 0.001 \\
\hline Food fortification policy & $0.36(0.20-0.64)$ & 0.001 \\
\hline \multicolumn{3}{|l|}{ Covariates } \\
\hline \multicolumn{3}{|l|}{ Age years median" } \\
\hline Second versus first quartile & $0.60(0.37-0.99)$ & 0.043 \\
\hline Third versus first quartile & $0.17(0.11-0.28)$ & $<0.001$ \\
\hline Fourth versus first quartile & $0.09(0.06-0.15)$ & $<0.001$ \\
\hline Sex male to female ratio ${ }^{\S}$ & $0.98(0.58-1.63)$ & 0.923 \\
\hline Mean BMIf & $0.39(0.32-0.48)$ & $<0.001$ \\
\hline Human Development Index ranking & $1.03(1.02-1.03)$ & $<0.001$ \\
\hline TB/HIV co-infection $\%$ of incident TB & $1.37(1.21-1.55)$ & $<0.001$ \\
\hline Type 2 diabetes prevalence $\%$ & $0.12(0.05-0.27)$ & $<0.001$ \\
\hline Alcohol use disorder prevalence above/below median & $0.55(0.35-0.87)$ & 0.010 \\
\hline \multicolumn{3}{|c|}{$\begin{array}{l}\text { p-values }<0.05 \text { are shown in bold. UV-B: ultraviolet } \mathrm{B} ; \text { BMI: body mass index. }{ }^{\#} \text { : anti-log values, as } \\
\text { outcome measures were log-transformed in analyses. " : the first quartile includes the lowest levels, and } \\
\text { the fourth quartile includes the highest levels. }{ }^{+} \text {:SD from mean monthly values }\left(\mathrm{J} \cdot \mathrm{m}^{-2} \text { ). }{ }^{\S} \text { : dichotomised as }\right. \\
\text { follows: }<100 \text { males per } 100 \text { females; and } \geqslant 100 \text { males per } 100 \text { females. } f: \text { categorised as follows: }<22.5 \\
\text { (category } 1 \text { ); } 22.5-25.0 \text { (category } 2 \text { 2); } 25.0-27.5 \text { (category } 3 \text { ); }>27.5 \text { (category } 4 \text { ) for univariate and } \\
\text { multivariate analyses. }\end{array}$} \\
\hline
\end{tabular}


TABLE 3 Primary analyses of the relationship between vitamin D proxies and tuberculosis incidence, and that between vitamin $\mathrm{D}$ proxies with TB incidence, adjusting for covariates

Primary analysis

Vitamin D proxy model

Fully adjusted model

Regression coefficient ${ }^{\#}(95 \% \mathrm{CI}) \quad$ p-value Regression coefficient ${ }^{\#}(95 \% \mathrm{Cl}$

p-value

\begin{tabular}{|c|c|c|c|c|}
\hline \multicolumn{5}{|l|}{ Mean UV-B exposure $\mathrm{J} \cdot \mathrm{m}^{-2} \cdot \mathrm{day}^{-1 \uparrow}$} \\
\hline Second versus first quartile & $0.99(0.64-1.54)$ & 0.962 & $0.70(0.45-1.07)$ & 0.098 \\
\hline Third versus first quartile & $0.71(0.41-1.21)$ & 0.205 & $0.55(0.34-0.91)$ & 0.020 \\
\hline Fourth versus first quartile & $0.42(0.21-0.83)$ & 0.013 & $0.22(0.12-0.43)$ & $<0.001$ \\
\hline Degree of urbanisation $\%$ & $0.98(0.97-0.99)$ & $<0.001$ & $1.00(0.99-1.01)$ & 0.917 \\
\hline \multicolumn{5}{|l|}{ Fish consumption $\mathrm{kg} \cdot$ capita $^{-1} \cdot \mathrm{year}^{-1 \uparrow}$} \\
\hline Second versus first quartile & $0.60(0.38-0.95)$ & 0.030 & $0.80(0.53-1.20)$ & 0.276 \\
\hline Human Development Index ranking & & & $1.03(1.02-1.03)$ & $<0.001$ \\
\hline Type 2 diabetes prevalence $\%$ & & & $0.68(0.34-1.37)$ & 0.278 \\
\hline
\end{tabular}

p-values < 0.05 are shown in bold. UV-B: ultraviolet B. " : anti-log values, as outcome measures were log-transformed in analyses; ": the first quartile includes the lowest levels, and the fourth quartile includes the highest levels; ${ }^{+}$: categorical variable.

The fully adjusted model explained $68.7 \%$ (95\% CI $67.1-70.3 \%)$ of the variance in country TB incidence, whereas this value was $63.9 \%$ (95\% CI 62.2-65.6\%) when UV-B exposure was excluded from the same model. The squared partial correlation coefficient for UV-B exposure in the fully adjusted model was 0.043. Thus, the proportion of the explained variation in per-country TB incidence that can be attributed to variation in UV-B exposure was $6.3 \%(0.043 / 0.687)$.

In the secondary national level analysis, the same covariates were identified as in the primary fully adjusted model (table 4). The reverse association between solar UV-B exposure and TB incidence, corrected for other vitamin D-proxies and covariates, was also retained in this analysis: countries in the highest UV-B quartile had $63 \%$ lower TB case notification rates compared to countries in the lowest UV-B quartile ( $\mathrm{p}=0.001$; table 4 ). The secondary, multilevel analysis included 475 countries and sub-national regions. In this model, mean BMI and TB/HIV co-infection were confounding covariates, in addition to type 2 diabetes prevalence and HDI ranking (table 4). The association between solar UV-B exposure and TB notification rates showed a similar trend, but with reduced magnitude in comparison to that of the primary analyses. Countries and sub-national regions in the highest solar UV-B quartile showed 29\% lower TB case notification rates as compared to countries and sub-national regions in the lowest quartile $(\mathrm{p}=0.057)$.

\section{Discussion}

In this ecological study exploring the relationship between solar UV-B exposure and global TB incidence, per-country $\mathrm{TB}$ incidence declined with increasing mean solar UV-B exposure, taking into account

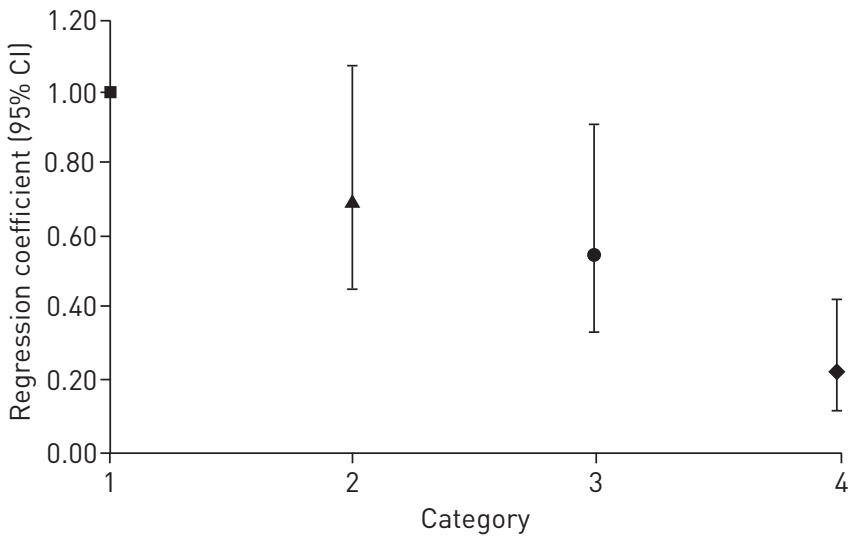

FIGURE 2 Association of tuberculosis (TB) incidence with annual solar ultraviolet B (UV-B) exposure, multivariable adjustment. $\beta$-coefficients for the association between mean TB incidence and quartiles of annual solar UV-B exposure in the fully adjusted linear regression model. Solar UV-B category 1 represents the reference category. $\beta$-coefficients shown are anti-log values, as mean TB incidence was log-transformed for analyses. Error bars depict the $95 \%$ confidence intervals. 
TABLE 4 Secondary analyses of the relationship between vitamin D proxies and covariates with tuberculosis (TB) case notification rates

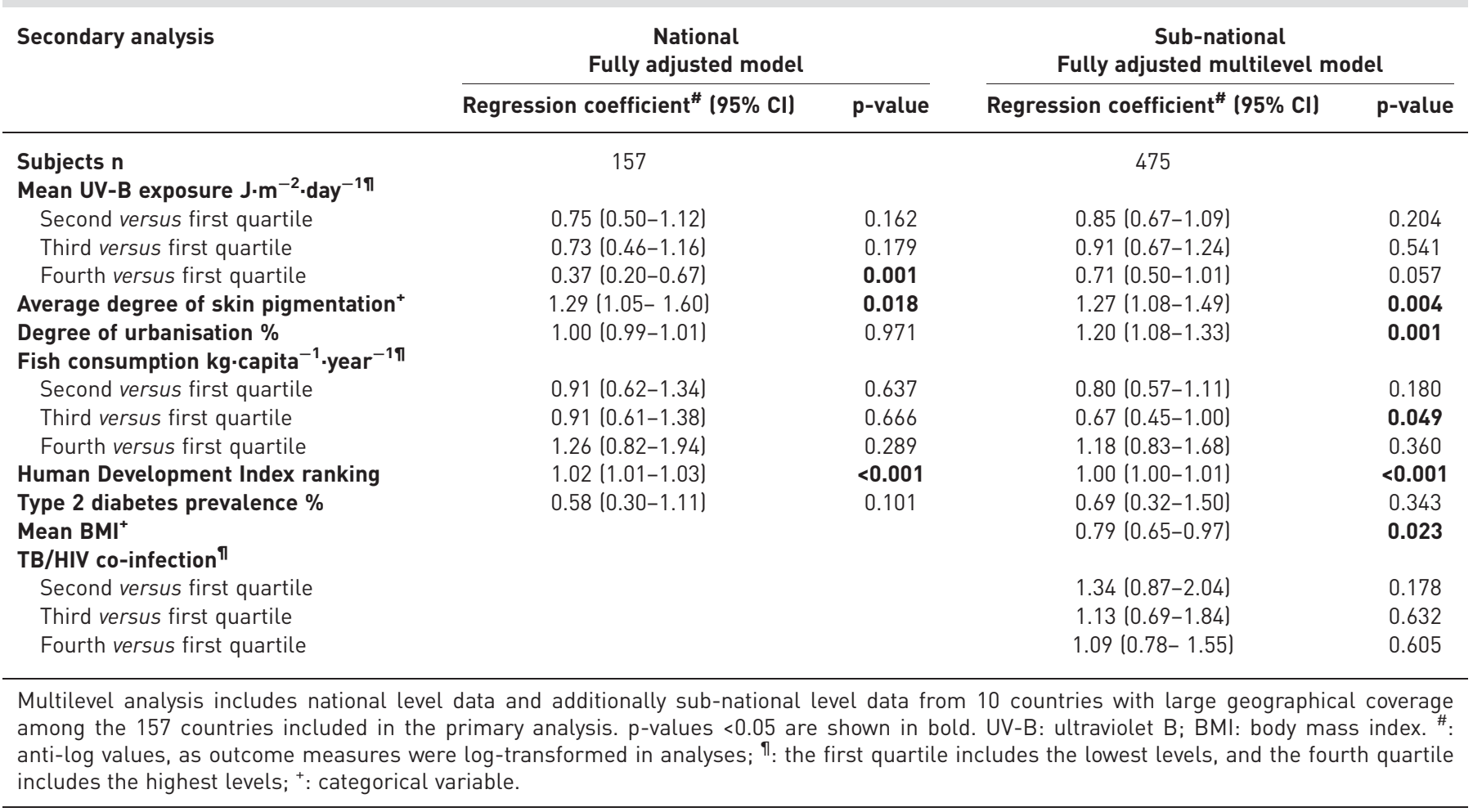

differences in skin pigmentation, urbanisation levels, fish consumption, type 2 diabetes prevalence, and HDI ranking. Countries in the highest mean solar UV-B exposure quartile had $78 \%$ lower TB incidence rates than countries in the lowest quartile, and differences in solar UV-B exposure accounted for $6.3 \%$ of the global variation in TB incidence explained by the model. When TB incidence was expressed as the per-country notification rate for bacteriologically confirmed, new pulmonary $\mathrm{TB}$, this negative association with solar UV-B exposure was largely retained, but showed less magnitude in an analysis that took into account sub-national regions from countries with large geographical latitude coverage.

Our findings corroborate the notion that variations in vitamin D status via sunlight exposure, moderated by skin pigmentation, could affect tuberculosis incidence [13, 27]. The effects of skin pigmentation in our analysis should only be interpreted in the context of sunlight exposure. Annual UV-B levels and skin pigmentation are highly correlated, and the direction of the association between UV-B levels and TB incidence changed from positive to negative after the addition of skin pigmentation to the model.

Several studies have demonstrated the temporality of low sunlight exposure/season, low vitamin D status, and TB incidence peaks, which is in line with our hypothesis $[28,29]$. However, in the present study, the seasonal variation in UV-B exposure did not significantly contribute to the vitamin D-proxy model. This might be because the unit of analysis was at the country level and tuberculosis incidence rates were averaged over each year within the given period. Seasonality of UV-B levels might result in notable variability in TB incidence within countries, but not necessarily lead to higher TB rates when drawing comparisons among countries.

Vitamin D supplementation in the treatment of active TB has shown little to no improvement in the clinical outcome and time to sputum culture conversion [7, 11,30]. Vitamin D prophylaxis has shown promise in a Mongolian population, in which fewer tuberculin skin test conversions were seen in the vitamin D supplementation group [10]. Vitamin D metabolites are predominantly involved in innate immunity, as they reduce initial bacterial invasion and support early clearance of tubercle bacilli $[7,9,10]$. Conversely, the role of vitamin $\mathrm{D}$ in the acquired immune response is mainly suppressive, as vitamin $\mathrm{D}$ metabolites downregulate pro-inflammatory cytokines and stimulate regulatory T-cells $[10,14,15]$. The resultant reduction in tissue damage would ameliorate symptoms, but might not necessarily clear infection any sooner $[7,19,30]$. Given these immunological properties, vitamin D sufficiency might be more beneficial for preventing progression to active disease than for improving the cure of active disease $[7,10,13]$. 
Our study has several limitations with regard to the available data. TB incidence as reported by the WHO might overestimate or underestimate true incidence, whereas notification rates do not take into account undetected or unreported TB patients. We could not validate our vitamin D-proxy model, as insufficient representative serum 25-hydroxyvitamin $\mathrm{D}$ data were available on a country-level worldwide scale. In particular, low UV-B exposure is correlated with low temperatures, and thus, with living indoors. Conceivably, the degree of urbanisation does not fully reflect indoor crowding, as in certain countries both urbanisation and temperature are associated with living indoors [31]. An indication that our findings reflect UV-B exposure rather than temperature, however, is the fact that several studies have reported lower TB incidence at higher altitudes where UV-B exposure is high, but temperatures are low [32, 33]. In addition, seasonal patterns of TB notification among immigrants in The Netherlands were consistent with increased disease progression, but not with increased transmission during the winter months [34]. Fish consumption data from the FAO website also included fish other than fatty fish, which might have limited the accuracy of this variable as a vitamin D proxy.

Other limitations relate to the ecological design of our study. There might have been residual confounding by factors such as skin coverage, low vitamin D diets, and vitamin D polymorphisms [35]. However, vitamin D-binding protein (DBP) genotypes for instance, appear to correlate with skin pigmentation; therefore, our analyses including skin pigmentation data should at least partially correct for a potential vitamin D-related association of DBP genotype with TB incidence [36-40]. Findings should not be directly extrapolated to individuals as the unit of analysis was at the country level instead of the individual level. Our study might be subject to ecological fallacy [41], as the country-level data did not provide information on individual levels of vitamin D proxies among TB patients and healthy individuals. This might have been especially important for countries with large variations in skin pigmentation, as opposed to countries with a relatively homogeneous distribution. Lastly, the multilevel secondary analysis included sub-national level data of mean UV-B and seasonality of UV-B exposure, TB case notification, skin pigmentation, urbanisation, male to female ratio, and HDI. As we were unable to find data for the remaining variables at the sub-national level, we assumed that these were homogeneous within countries. This assumption could have masked important sub-national differences, particularly for TB/HIV co-infection and BMI, therefore potentially resulting in residual confounding. Overall, our results might provide supportive evidence for hypotheses concerning vitamin $\mathrm{D}$ and $\mathrm{TB}$ incidence.

Despite these limitations, ecological studies do have the merit of providing stronger evidence with the use of population context data of individual characteristics, as opposed to individual level data regarding risk factors [35]. Dietary assessments provide an excellent example: within-person variability in diet is generally greater than variability at the population level [41]. In addition, solar UV-B levels were measured with great precision and validity, which strengthens our findings [20]. Furthermore, ecological studies are an efficient way of exploring relationships on a global level, especially when based on publicly available data $[41,42]$.

In conclusion, when taking into account other determinants of vitamin D levels (notably skin pigmentation), solar UV-B exposure is evidently an independent determinant of TB incidence at the country level worldwide. This association remained robust with regard to the direction and magnitude, irrespective of the outcome measure (TB incidence or notification), although the magnitude of the association was less when sub-national rather than national data were considered. These findings suggest that variation in solar exposure is a plausible explanation for part of the previously unexplained global variation in TB incidence. Future studies should determine the influence of pre-existing vitamin D sufficiency as a factor of immunocompetence when exploring the relationship between vitamin D status and tuberculosis. For instance, migrant studies exploring vitamin D prophylaxis might provide useful insights. Furthermore, a study that focuses on absolute and relative declines in serum 25-hydroxyvitamin $\mathrm{D}$ levels during different seasons and is associated with ethnicity (as a proxy for skin type), vitamin D-binding protein genotypes, anthropometric measures (such as BMI and central adiposity), nutritional patterns and daytime activity levels could provide useful insights into the interactions between various vitamin $\mathrm{D}$ proxies and vitamin $\mathrm{D}$ status.

\section{Acknowledgements}

We wish to thank Patricia Bartholomay (Tuberculosis National Control Program, Secretariat of Health Surveillance, Ministry of Health of Brazil, DF, Brazil), Anete Trajman (Federal University of Rio de Janeiro, Rio de Janeiro, Brazil), Nguyen Viet Nhung and Nguyen Binh Hoa (Viet Nam National Tuberculosis Control Program), Soledad Beltrame (National Tuberculosis and Lepra Control Programme, Buenos Aires, Argentina), Zhao Yong (Dept. of Nutrition and Food Hygiene, School of Public Health and Management, Chongqing Medical University, Chongqing, China), Sergey Sterlikov (Federal TB Monitoring Center, Federal Research Institute for Health Organization and Informatics, Moscow, Russian Federation), Puneet Dewan (Senior Program Officer for TB, Bill and Melinda Gates Foundation, New Delhi, India), Richard Menzies (Montreal Chest Institute, Montreal, Canada), and Gunta Dravniece (Senior Consultant, KNCV Tuberculosis Foundation, The Hague, Netherlands) for their assistance in the collection of sub-national tuberculosis case 
notification data. Lastly, we thank Ente Rood (Environmental epidemiologist, KIT Biomedical Research, Amsterdam) and Mirjam Bakker (Senior epidemiologist, KIT Biomedical Research, Amsterdam) for their guidance in using the Geographical Information System software.

\section{References}

1 Glaziou P, Sismanidis C, Floyd K, et al. Global epidemiology of tuberculosis. Cold Spring Harb Perspect Med 2015; 5: a017798.

2 World Health Organization (WHO). Global Tuberculosis Report 2015. Geneva, WHO Press, 2015. http://apps. who.int/iris/bitstream/10665/191102/1/9789241565059_eng.pdf?ua=1

3 Lin PL, Flynn JL. Understanding latent tuberculosis: a moving target. J Immunol 2010; 185: 15-22.

4 Barry CE, Boshoff $\mathrm{HI}$, Dartois V, et al. The spectrum of latent tuberculosis: rethinking the biology and intervention strategies. Nat Rev Microbiol 2009; 7: 845-855.

5 Shankar EM, Vignesh R, Ellegård R, et al. HIV-Mycobacterium tuberculosis co-infection: a "danger-couple model" of disease pathogenesis. Pathog Dis 2014; 70: 110-118.

6 Lönnroth K, Jaramillo E, Williams BG, et al. Drivers of tuberculosis epidemics: the role of risk factors and social determinants. Soc Sci Med 2009; 68: 2240-2246.

7 Ralph AP, Ralph AR, Lucas RM, et al. Vitamin D and solar ultraviolet radiation in the risk and treatment of tuberculosis. Lancet Infect Dis 2013; 13: 77-88.

8 Davies P. A possible link between vitamin D deficiency and impaired host defence to Mycobacterium tuberculosis. Tubercle 1985; 66: 301-306.

9 Verrall AJ, Netea MG, Alisjahbana B, et al. Early clearance of Mycobacterium tuberculosis: a new frontier in prevention. Immunology 2014; 141: 506-513.

10 Ganmaa D, Giovannucci E, Bloom BR, et al. Vitamin D, tuberculin skin test conversion, and latent tuberculosis in Mongolian school-age children: a randomized, double-blind, placebo-controlled feasibility trial. Am J Clin Nutr 2012; 96: 391-396

11 Wejse C, Gomes VF, Rabna P, et al. Vitamin D as supplementary treatment for tuberculosis: a double-blind, randomized, placebo-controlled trial. Am J Respir Crit Care Med 2009; 179: 843-850.

12 Bendik I, Friedel A, Roos FF, et al. Vitamin D: a critical and essential micronutrient for human health. Front Physiol 2014; 5: 1-14.

13 Ustianowski A, Shaffer R, Collin S, et al. Prevalence and associations of vitamin D deficiency in foreign-born persons with tuberculosis in London. J Infect 2005; 50: 432-437.

14 Chun RF, Adams JS, Hewison M. Immunomodulation by vitamin D: implications for TB. Expert Rev Clin Pharmacol 2011; 4: 583-591.

15 Harishankar M, Afsal K, Banurekha VV, et al. 1,25-Dihydroxy vitamin D3 downregulates pro-inflammatory cytokine response in pulmonary tuberculosis. Int Immunopharmacol 2014; 23: 148-152.

16 Kwan C, Ernst JD. HIV and tuberculosis: a deadly human syndemic. Clin Microbiol Rev 2011; 24: 351-376.

17 Dye C, Lönnroth K, Jaramillo E, et al. Trends in tuberculosis incidence and their determinants in 134 countries. Bull World Health Organ 2009; 87: 683-691.

18 Lonnroth K, Williams BG, Cegielski P, et al. A consistent log-linear relationship between tuberculosis incidence and body mass index. Int J Epidemiol 2010; 39: 149-155.

19 World Health Organization. Global Tuberculosis Report 2013. Geneva, World Health Organization, 2013.

20 Beckmann M, Václavík T, Manceur AM, et al. A global UV-B radiation dataset for macroecological studies. Methods Ecol Evol 2014; 5: 372-383.

21 Tanskanen A, Krotkov N, Herman J, et al. Surface ultraviolet irradiance from OMI. IEEE Trans Geosci Remote Sens 2006; 44: 1267-1271.

22 Food and Agriculture Organization of the United Nations. Data structure, concepts and definitions common to FAOSTAT and CountrySTAT framework. http://www.countrystat.org/resources/metadata_en.pdf Date last updated: June 2010. Date last accessed: March 3, 2015.

23 Liberato SC, Pinheiro-Sant'Ana HM. Fortification of industrialized foods with vitamins. Rev Nutr Campinas 2006; 19: 215-231.

24 Hackett CP, Grim BJ. The global religious landscape: a report on the size and distribution of the world's major religious groups as of 2010. Washington, Pew Research Center, 2012; pp. 45-50.

25 Prüss-üstün A, Zeeb $\mathrm{H}$, Mathers $\mathrm{C}$, et al. Solar ultraviolet radiation - global burden of disease from solar ultraviolet radiation. Geneva, World Health Organization, 2006.

26 Holick MF. Vitamin D and health: evolution, biologic functions, and recommended dietary intakes for vitamin D. In: Holick MF, ed. Vitamin D: Physiology, Molecular Biology, and Clinical Applications. 2nd Edn. New York, Springer Science \& Business Media, 2010; p. 27.

27 Zammarchi L, Bartalesi F, Bartoloni A. Tuberculosis in tropical areas and immigrants. Mediterr J Hematol Infect Dis 2014; 6: e2014043.

28 MacLachlan JH, Lavender CJ, Cowie BC. Effect of latitude on seasonality of tuberculosis, Australia, 2002-2011. Emerg Infect Dis 2012; 18: 1879-1881.

29 Visser DH, Schoeman JF, Van Furth AM. Seasonal variation in the incidence rate of tuberculous meningitis is associated with sunshine hours. Epidemiol Infect 2013; 141: 459-462.

30 Martineau AR, Timms PM, Bothamley GH, et al. High-dose vitamin D3 during intensive-phase antimicrobial treatment of pulmonary tuberculosis: a double-blind randomised controlled trial. Lancet 2011; 377: 242-250.

31 Naranbat N, Nymadawa P, Schopfer K, et al. Seasonality of tuberculosis in an Eastern-Asian country with an extreme continental climate. Eur Respir J 2009; 34: 921-925.

32 Olender S, Saito M, Apgar J, et al. Low prevalence and increased household clustering of Mycobacterium tuberculosis infection in high altitude villages in Peru. Am J Trop Med Hyg 2003; 68: 721-727.

33 Mansoer JR, Kibuga DK, Borgdorff MW. Altitude: a determinant for tuberculosis in Kenya? Int J Tuberc Lung Dis 1999; 3: 156-161.

34 Korthals Altes H, Kremer K, Erkens C, et al. Tuberculosis seasonality in the Netherlands differs between natives and non-natives: a role for vitamin D deficiency? Int J Tuberc Lung Dis 2012; 16: 639-644. 
35 van Schoor NM, Lips P. Worldwide vitamin D status. Best Pract Res Clin Endocrinol Metab 2011; 25: 671-680.

36 Constans J, Lefevre-Witier PH, Richard P, et al. Gc (vitamin D binding protein) subtype polymorphism and variants distribution among Saharan, Middle East, and African populations. Am J Phys Anthropol 1980; 52: 435-441.

37 Gozdzik A, Zhu J, Wong BYL, et al. Association of vitamin D binding protein (VDBP) polymorphisms and serum 25(OH)D concentrations in a sample of young Canadian adults of different ancestry. J Steroid Biochem Mol Biol 2011; 127: 405-412.

38 Nielson CM, Jones KS, Chun RF, et al. Free 25-hydroxyvitamin d: impact of vitamin d binding protein assays on racial-genotypic associations. J Clin Endocrinol Metab 2016; 101: 2226-2234.

39 Holick MF. Bioavailability of vitamin D and its metabolites in black and white adults. N Engl J Med 2013; 369: 2047-2048.

40 Chun RF, Peercy BE, Adama JS, et al. Vitamin D binding protein and monocyte response to 25-hydroxyvitamin D and 1,25-dihydroxyvitamin D: analysis by mathematical modeling. PLoS One 2012; 7: e30773.

41 Levin KA. Study design VI - Ecological studies. Evid Based Dent 2006; 7: 108.

42 Morgenstern H. Ecologic studies in epidemiology: concepts, principles, and methods. Annu Rev Public Health 1995; 16: 61-81. 